\title{
Index of Ancient and Medieval Authors ${ }^{1}$
}

Aeg. Paris.

Evang.

$1995-1996 \quad 502$

1997-2002 502

Alb. Muss.

Fam.

$X .4 .1483$

Ep.

IV.61-62 $485 f$.

Alc. Avit.

Epist. ad Apollin.

$13 f ., 27$

Epist. 25bis.

368

Prol.

SHG 28

\section{SHG}

1.24-29 59

I.211-217 31

I.218-224 58

I.223-227 59

$1.238-246 \quad 34$

I. $251-253 \quad 35$

1.29033

I.293-294 33

I.297-298 33

II.31-32 140

II.154 68

III.12-15 133

III. $213-27860 \mathrm{f}$.

III.334-341 70 $\begin{array}{lc}\text { IV. } 386-387 & 68 \\ \text { IV. } 516-521 & 33\end{array}$

Virgin.

417-440 146

$421 \quad 133$

$422 \quad 133$

$423 \quad 135$

$425 \quad 138$

$426 \quad 140$

$427 \quad 140$

$430-440 \quad 140$

$430 \quad 140$

431140 bis

433140

434140 bis

438140

$440 \quad 140$

Alex. Hal.

Summa theol.

I.1 475, 476

Ambr.

Exc. Sat.

II.78-79 297

Helia

3.423

lacob

I.1.4 133

in Luc.

V.107 64

VIII.18 65

1 Only explicit quotations of texts are taken into consideration; the numbers correspond to the pages, they are in Italic if the quote is in the notes. 
Nab.

$\begin{array}{lc}1.1 & 64 \\ 1.3 & 65 \\ 2.4 & 65 \\ 5.26 & 65\end{array}$

parad.

$15.7 \quad 221$

Anth. Lat.

I, $524 \quad 36$

\section{Apocalypsis Goliae}

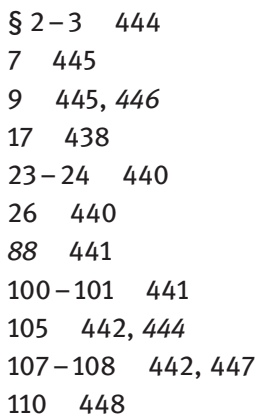

\section{Arator}

ad Vigil.

15-16 81

$19-20 \quad 13$

act.

$1.69-83 \quad 77$

I.83-84 76

I. $261-262 \quad 47$

I.297-301 84

$1.634-64284$

I.797 47

I.796-797 48

II. $40-4277$

II.43-44 77

II. $216-21878$

II. $262-273 \quad 85$

II.274 86

II.532 93

II.831-832 80

II.839-844 81
II.871-879 82

II.890-891 75

II.1233-1245 78

Vig.

$17-22 \quad 75$

Aug.

civ.

VI.6 124

XVIII.14 482

coll. Don.

III.2.51 123

conf.

X.27.38 483

gen. c. Manich.

460

gen. ad litt.

XII.11 443

XII.34 444

in psalm.

30.2.14. 85

serm.

2.74

$19.4 \quad 497$

$41.4 \quad 502$

$97.14 \quad 133$

122.1141

$296.9 \quad 82$

Avien.

Arat.

292-294 20

338-342 22 
Bacch.

Od.

5.56-84 $249 \mathrm{f}$.

Bruno de Segni

Comm. in Luc. p. II

PL 165.422CD 502

PL 165.425 A 501

De Cain, P. Bodm.

33.17-19 196

Call.

Hymn. Apoll.

$2.10 \quad 263$

Carm. de ressur.

292-332 398

Cic.

de orat.

II.15.62-63 3

part.

9.324

Clem. Al.

Protr.

$12.120 .2 \quad 155$

Strom.

I.28.178 176

IV.25.162 153

Col. Salut.

Lab. Herc.

I.9.25-28 485

\section{Commod.}

instr.

II.10.9-11 136

\section{Const. apost.}

V.7.12 50

\section{Cyr. Al.}

In Io.

IV.5 163f., 166, 167

De Abel. verbis, P. Bodm.

35.15-18 197

De Abraham, P. Bodm.

30.1-3 195

20-21 195

De iustis, P. Bodmer

31.111-119 193

Drac.

Laud.

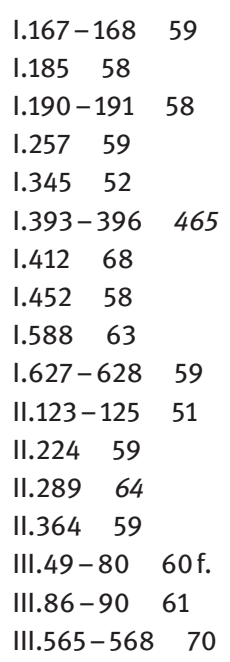

Satisf.

1564 
Eleg. in Maecen.

$1.23-24 \quad 20$

Eur.

Bacc.

$\begin{array}{lc}274-285 & 151 \\ 311-313 & 157 \\ 343-346 & 157 \\ 471-472 & 153 \\ 479-480 & 157 \\ 482-484 & 157 \\ 489-490 & 157 \\ 655-656 & 157 \\ 773-774 & 152\end{array}$

Euseb.

Praep. Evang.

XI.5 223

Euseb. Emes.

Serm.

11.2136

Faustin.

Fid.

$5.20 \quad 330$

Greg. Illib.

Fid.

$\begin{array}{cc}226.186 & 331 \\ 226.209 & 331 \\ 226.220 & 331 \\ 227.227 & 331 \\ 226.234 & 331 \\ 226.247 & 331 \\ 228.262 & 332\end{array}$

Hom.

VI.19 345
Greg. Naz.

Poem.

I.1.16.5-6 282

I.1.16.10 278

I.1.16.17 278

I.1.16.21 282

I.1.16.29-30 282

I.1.20.3 278

I.1.20.4 278

I.1.20.5 278

I.1.20.13 279

I.1.20.19-20 280

I.1.20.25-26 279

I.1.20.29-30 279

I.1.21.5 279

I.1.21.6 277

I.1.21.7 277

I.1.21.9-10 280

I.1.21.13-14 $279 \mathrm{f}$.

I.1.21.17 280

I.1.22.20 282

I.1.22.9-10 277

I.1.22.13 280

I.1.23.7 280

I.1.23.8 280

I.1.34.77 279

I.1.34.83 279

II.1.2.1-14 $262 \mathrm{f}$.

II.1.2.58-69 $264 \mathrm{f}$.

II.1.2.149-155 268

II.1.2.204-208 269

II.1.15.21-30 283

II.1.19.17-22 284

II.1.19.25-31 285

II.1.34.71-72 273

II.1.34.77-78 273

II.1.34.83 273

\section{Heptateuchos}

gen.

$1-2 \quad 458$

$15-16 \quad 115$

$20 \quad 116$

$50-51 \quad 108$

$66 \quad 106$

$81 \quad 121$

120-121 120

$140 \quad 119$ 
147116

154119

$162-163119$

$188-190 \quad 113$

$197-198 \quad 112$

243-248 110

290116

290-292 110

293-294 116

306-307 116

$316-317 \quad 107$

324120

326107

361-362 123

365120

463-466 121

592112

593117

596108

$597 \quad 117$

620117

745-746 108

884110

903117

904109

935109

1081-1083 112

1141-1142 115

1147-1149 117

1172-1174 113

$1200 \quad 114$

1236-1237 113

1240-1241 118

1255-1257 118

1312109

$1400 \quad 110$

1418-1419 109

1433-1437 123

los.

$49 \quad 116$

Hier.

Epist.

107.4356 in Matth.

III.21.6 134

III.21.18 132, 133

III.21.19 134

vir. ill. $84 \quad 42$

Hilar. (?)

hymn. de Christo

$31-32 \quad 365$

Hilar.

Matth.

VIII.8 47

Himn. Hisp. 1 Castro Sánchez

1411

1-40 406f.

12411

$18-20 \quad 408$

$20 \quad 410$

21-24 408

$29-30 \quad 407$

$34-36 \quad 408,410$

25 Castro Sánchez

1-32 $412 \mathrm{f}$.

3413

6-8 413

$13-16 \quad 413$

$17-20 \quad 413 \mathrm{f}$.

$22 \quad 414$

25-28 414

190 Castro Sánchez

$1-50 \quad 417 \mathrm{f}$.

10419

$10-15 \quad 418$

$15 \quad 419$

$16-20 \quad 419$

21-27 419

$22 \quad 419$

3419

$34-35 \quad 420$ 
Hom.

II.

I.68-70 109

XVIII.483-484 195

Od.

IV.17-19 232

XI.34-35 247

XI.36-37 247

XI.42-50 248

XI.164-165 248

XI.205-208 248

XI.475-476 248

XI.636-640 249

Hor.

Ars

9-10 14

151-152 15

Serm.

I.2.131 299

I.2.134 299

Hugo de Sanct. Vict.

Sacr.

Prol. 6527

Ioh. Gers.

Decl. comp.

6.184-185 G. $\quad 530$

Gratia tibi

276 G. 528

Josephina

II.411-424 530

III.855-860 528f.

IX.2278-2287 530

X.2504-2507 530
Sens. litt. Sacr. Script.

3.354 G. $\quad 533$

3.355 G. $\quad 532$

Super Cant. Cant.

565 G. $\quad 512$

576 G. $\quad 511,515$

577 G. $\quad 513,515 f$.

579 G. $\quad 523$

$581 \mathrm{G} . \quad 514$

590 G. $\quad 516$

$591 \mathrm{G} . \quad 519$ f., 522

Ioh. Parv.

lust. Ducis Burg.

$28-30 \quad 531$

Ioh. Scott.

Praed.

$9476 \mathrm{f}$.

Trans. de deo praed.

$10 \quad 477$

Sup. Ier.Cael. Sancti Dionysii

PL 122.146B-C 477

Iren.

Adv. Haer.

IV.34.4 342, 344

Isid.

In los.

VII.1 95

Itin. Anton. Plac. Rec. A

$17 \quad 142$ 
Iuv.

Sat.

$1.27-29 \quad 63$

1.7964

1.8564

$1.147-14963$

$6.268-271 \quad 114$

$6.284-285114$

14.10-14 113

$14.18-20 \quad 113$

$14.303-304 \quad 65$

\section{luvenc.}

Praef.

$15-18 \quad 13$

1942

Evang.

I.323-325 18

I.344 135

$1.631-63319$

1.653135

II.89-94 51

II.115-117 132

II.119 92

II.24293

II.753 135

III.653-674 145

III.662-663 136

III.663 137

III.671 138

IV.369-370 299

IV.376-379 296

IV.392-393 299

IV.394-395 297

IV.394-396 296

IV.400 138

IV.630-631 142

Lact.

Inst.

I.5.8-10 329, 333

II.8.3 333

IV.6.1 333

IV.6.9 333

IV.7.1 333

\section{Laudes Domini}

7-35 291-293

15-16 295

$25 \quad 296$

112-115 46

Lucan.

VI.753 299

Lucr.

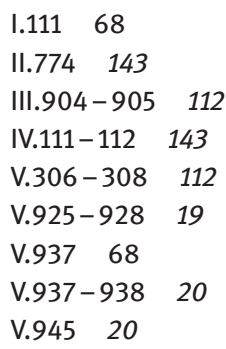

Man. Phil.

Ps. 32 versio $A$.

1-4 231

Ps. 34 versio $A$.

$8-11 \quad 232$

Ps. 102

36-43 233

Mar. Vict.

Aleth.

II.1-5 14

II.195-198 16

\section{Max Taur.}

Serm.

38.2.3.4 344

Nican.

Ther.

957-958 286 


\section{Nonn.}

Dion.

VII.82-88 152

VII.94-96 152

XVII.373 180

Par.

I.129-130 180

I.135 180

II.12-20 153

II. $35-38 \quad 154$

II. 39154

V.110 180

VI.4 181

VI.122-123 170

VII.7-8 161

VII.8-9 162

VII.13-15 162

VII.19 162

VII.20 162

VII.21 162

VII.22 163

VII.24-25 163

VII.26 163

VII.27-28 163

VII.30 $163 \mathrm{f}$.

VII.31 161

VII.37 161

VII.138 164

VII.41-45 155

VII.46-49 164

VII.53 165

VII.55-57 166

VII.65 166

VII.67-68 166

VII.119 166

VII.166 166

VII.64-68 155

VII.72-73 167

VII.79-80 165

VII.85 167

VII.89 167

VII.90 167

VII.103 168

VII.104 169

VII.114 165

VII.117-119 168

VII.12 165

VII.128 169
VII.138-139 169

VII.139 169

VII.143-145 169

VII.143-147 170

VII.154-155 168

VII.171 165

VII.172-182 156

VII.17 156

VII.181-182 168

VII.18 170

VII.190-193 170

X.133 178

XI.89 182

XI.96-102 155

XI.155-170 298

XI.159 299

XI.185-206 158

XIII.30 179

XIII.85-89 181

XIII.158-160 182

XVIII.95-96 179

Opt. Porph.

carm.

$16.23-25 \quad 80$

\section{Orat. Wisig. Vives}

9410

35409

$63 \quad 410$

$127 \quad 410$

$536 \quad 414$

\section{Ferotin}
c. 15-16 409
c. $490 \quad 410$
c. $576 \quad 410$
c. $875 \quad 410$

Orig.

in lesu Nave hom.

$6.4 \quad 95$

7.195 
Ov.

am.

III.8.33-34 19

III.11.47 468

ars

III.723 108

fast.

I.185 134

IV.747-755 121

met.

I. $21-22 \quad 458$

I.71 464

I.128-130 22

III.725-727 118

VII.502 92

VIII.674 134

XI.1-2 138

XI.339-341 116

XIV.124 468

XIV.338-340 138

trist.

I.1.35 92

Paul. Nol.(?)

carm. 6(= Laus Iohannis)

229-254 17

$229 \quad 18$

$247 \quad 23$

$252 \quad 23$

Paul. Nol.

carm. 19 (= Nat. 11)

14-19 366

$54-56 \quad 367$

$76-84 \quad 367$

carm. 20 (=Nat. 12)

$28-32 \quad 15$ carm. 24 (ad Cyth.)

\begin{tabular}{|c|c|}
\hline $495-498$ & 352 \\
\hline $499-506$ & 351 \\
\hline $513-516$ & 356 \\
\hline $517-524$ & 352 \\
\hline $525-528$ & 350 \\
\hline $541-588$ & $361 \mathrm{f}$. \\
\hline $639-642$ & 357 \\
\hline $695-696$ & 358 \\
\hline $701-712$ & 358 \\
\hline $707 \quad 359$ & \\
\hline $710 \quad 359$ & \\
\hline $749-756$ & 355 \\
\hline $753 \quad 357$ & \\
\hline $827-830$ & 356 \\
\hline $889-890$ & 353 \\
\hline $891-893$ & 359 \\
\hline $895 \quad 360$ & \\
\hline 896360 & \\
\hline carm. 25 & $=$ Epith. Iul.) \\
\hline $236-237$ & 353 \\
\hline carm. 31 (= & = obit. (els.) \\
\hline $465 \quad 68$ & \\
\hline $470-472$ & 67 \\
\hline $473-474$ & 68 \\
\hline $488 \quad 68$ & \\
\hline $495-498$ & 68 \\
\hline $617-618$ & 359 \\
\hline
\end{tabular}

Paul. Petr.

Mart.

$\begin{array}{ll}\text { I. } 232-234 & 81 \\ \text { II. } 141-148 & 19\end{array}$

Pers.

$3.86-87 \quad 112$

Petr. Abael.

Theol. Christ.

PL 178.1207C 478 
Petr. Crys.

Sermo

$65 \quad 298$

Petr. Riga

Aurora

$2053-2056 \quad 499$

Evang.

$33-34 \quad 466$

61465

$129-134 \quad 468$

409-410 454

2889-2890 456

2893-2896 456

2895-2896 456

Gen.

$7-8 \quad 459$

21-22 460

99462

163-166 463

$304 \quad 464$

306464

$310 \quad 464$

323-324 469

361-362 465

Phipp. Harv.

Inst. cler.

III.45 482

Poetae lat. aev. Carol.

II 254.21-24 431

Proba

Cento

173-174 317

177-178 317

195-205 315

276-277 22

290-293 22
299-302 22

580-599 310

Prud.

apoth.

$207 \quad 327$

212-214 327

264-267 328

$672-674 \quad 46$

$752-766 \quad 300$

938-939 68

cath.

3.1-5 330, 340

4.7-9 327

9.10-12 329

$9.31-3352$

$9.40-4253$

$9.6952,53$

11.17-32 329f.

$12.37-40 \quad 328$

12.125411

c. Symm.

I. $325-327 \quad 327$

II.85-90 324

II. $87 \quad 324$

II. $87-88 \quad 325$

II.91-107 324

II.120-124 325

Ditt.

$183 \quad 54$

ham.

27919

$282-284 \quad 19$

395-397 140

76364

$942-94368$

Perist.

$2.30 \quad 411$

psych.

$629-630 \quad 140$ 
Ps.-Apol.

Met. psalm.

Protheoria 15-16 227

18-19 227

29-32 227

Ps. 32.1-4 231

Ps. 46.8-14 232

Ps. 102.38-44 233

\section{Ps.-Cypr.}

resurr.

$300 \quad 68$

\section{Quint.}

Inst.

VI.2.32 52

\section{Raban.}

Hom.

II.77 502

Rhet. Her.

I.9.16 4

Rupert. Tuit.

Comm. Job

168.1131B 501

Sall.

Cat.

$5.8 \quad 22$

Sedul.

Carm. Pasch.

1.1268

I.17-18 13

$1.23 \quad 13$

I.26 8,13

$1.30-35 \quad 47$

$1.99-100 \quad 70$

1.3609
II.94. 7

II.97 10

II.168-171 10

III.33-39 48

III.148-151 49

III. $273 \quad 47$

IV.42-56 145

IV.49 135

IV.210-221 $95 \mathrm{f}$.

IV.213-215 $95 \mathrm{f}$.

IV. $222-225 \quad 6$

IV. 251-270 $98 \mathrm{f}$.

IV. $255-2567,99$

IV.263-264 10, 99

IV.287 299

V.71 7

V.77-78 7

V.166-167 7

V.168-169 7

V.180-181 7

V.420 9

Epist.

$1.12 .4-5 \quad 8$

Op. Pasch.

II.4 9, 10

II.7 10

II.13 10

II.1410

III.14 10

IV.5 138

V.8 10

V.10 9

V.17 10

V.22 10

V.37 8

Ser.

med.

$974 \quad 134$

Serv.

ad Aen.

I, $159 \quad 37$ 
ad ecl.

Synes.

1.34130

Hymn.

Severus

8.1-71 241f.

in Evang.

Theophylact.

VIII.108 94

VIII.119-136 90

VIII.137-138 90

VIII.137-153 90

VIII.145-146 92

VIII.149-151 93

IX.81-93 100

IX.93 129

IX.93-140 $146 \mathrm{f}$.

IX.9694, 131

IX.104 138

IX.106 94

IX.107 141

IX.119 141

IX.137 143

IX.137-149 101

IX.138-139 143

IX.140 143

IX.163-180 97f

IX.163 98

IX.164 98

IX.251 93

Sidon.

Enarr. in evang.

Marc. $232 \quad 127$

Thomas Aq.

Comm. sent. Petr. Lomb.

I.1.1.3 $479-480$

I.1.1.6 481

I.1.1.15 481

Super meteora

II.1.3. 481

Sent. Metaph. I

II.11.3 481

Ven. Fort.

Carm.

V.2.1-20 $373 \mathrm{f}$.

VIII.3.79-80 377

VIII.3.173 377

Carm.

$11.126 \quad 135$

Ven. Fort. (?)

in laud. sanctae Mariae

Stat.

$267-270 \quad 382$

275-296 382

Th.

V.445-446 132

X.674 135

Verg.

Aen.

Sulp. Sev.

I.216 110

1.30494

Mart.

I.305 117

10.819

I.503-504 120

I.701 313

1.706313

II. $252-254 \quad 120$

II.634 108 
II.730 94

III.205-206 107

IV.453-454 118

V.40-41 109

V.84-93 $316 \mathrm{f}$.

V.615-616 110

VI.255 108

VI.258 263

VI.536-537 108

VII.68-70 107

VII.254 117

VII.539 119

VII.808-811 116

VIII.8-9 115

VIII.69-71 78

VIII.84 110

VIII.90 109

VIII.324-327 22

IX.144 106

IX.393 117

XII.172 459

XII.359-361 120

XII.664 119

XII.689-692 119

XII.173 313 ecl.

1.51-52 117

$4.7 \quad 461$

$5.38-39 \quad 120$

georg.

I.19 345

1.78442

II. $458-460 \quad 18$

III.242-244 110

IV.478-479 116

IV.39 109

Versus memoriales (BHL 654k)

$387 \mathrm{f}$.

Vincent. Bell.

Spec. Doctr.

1.13476

Vigil.

Epist. ad Auxanium

381 
